\title{
Síndrome cerebeloso secundario a granulomas de fosa posterior y tuberculosis multisistémica en un paciente con SIDA
}

\author{
Claudio Navarrete A., Mónica Rosas K., David Sáez M., \\ Eugenio Tenhamm F. y Jorge Nogales-Gaete
}

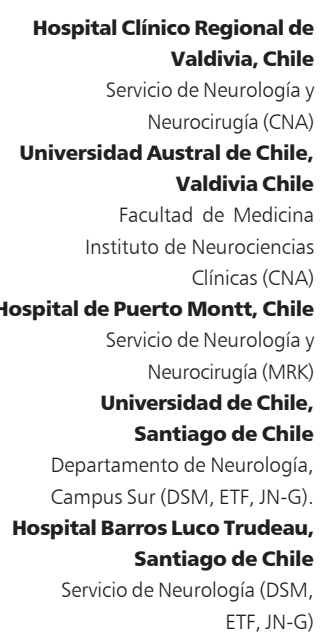

Recibido: 8 enero 2007 Aceptado: 10 enero 2008

Correspondencia a: Claudio Navarrete Asenjo cnavarreteasenjo@yahoo.es

\section{Cerebelous syndrome by infratentorial brain tuberculomas and mutisystemic tuberculosis in AIDS patient}

We present a clinical case of a patient who consulted for a cerebelous syndrome and diplopia associated to $10 \mathrm{~kg}$ weight loss in six months and multiple adenopathies. Cerebral imagenology study evidenced the presence of granulamatous masses at the cerebellum and midbrain level, there were no cerebrospinal fluid abnormalities. Systemic tuberculosis (TB) diagnosis was done based in finding of acid-fast bacilli positive staining in urine and lymph node biopsy. HIV infection was documented by ELISA serology. CD4 cell count was $590 \mathrm{cell} / \mathrm{mm} 3$, classifying the case a AIDS C1 stage. TB was treated according to national guidelines leading to good clinical and imagenology evolution. The clinical case scenario of infratentorial tuberculomas without meningitis as a first manifestation of TB and AIDS is unusual, generating our interest in reporting this case.

Key words: Tuberculosis, tuberculoma, AIDS.

Palabras clave: Tuberculosis, tuberculoma, SIDA

\section{Introducción}

$\mathrm{L}$ a TBC es un problema de salud pública mundial que representa la segunda causa de muerte por enfermedades infecciosas ${ }^{1-3}$. Es causada por Mycobacterium tuberculosis; con menos frecuencia otras micobacterias, como M. bovis, M. africanum, $M$. ulcerans, y en hospederos inmunocomprometidos también $M$. avium intracellulare, pueden ocasionar un cuadro similar ${ }^{3,4}$.

En los últimos años la TBC se ha agudizado en los países no industrializados y ha reaparecido en los desarrollados, asociado a la pandemia del SIDA; junto a ello, ha aumentado la resistencia a terapia antituberculosa ${ }^{3}$. En Chile, la TBC ha declinado progresivamente en la última década, a diferencia de países con nivel de desarrollo similar ${ }^{5,6}$. Si bien, puede afectar cualquier órgano $0^{3,4}$, el compromiso del sistema nervioso central (SNC) es el de mayor gravedad ${ }^{7,8}$.

Presentamos el caso clínico de un paciente con TBC sistémica, que debutó con síntomas neurológicos y en el que se demostró la presencia de tuberculomas parenquimatosos encefálicos, sin compromiso meníngeo.

\section{Caso Clínico}

Varón de 36 años, soltero, con educación media, obrero. Con antecedentes de hipertensión arterial sin tratamiento, consumo de cigarrillos, alcohol y drogas ilícitas (anfetaminas y pasta base). Sin antecedentes de hemotransfusiones. Tuvo un episodio de neumonía intersticial en 1997, realizándosele baciloscopias de expectoración que resultaron negativas. A inicios del año 2005 se controló en reumatología por poliartralgias y eritema de extremidades inferiores (con regresión espontánea), constatándose discreto ensanchamiento mediastínico en una radiografía de tórax.

El cuadro clínico actual se inició en marzo del 2005 con baja de peso sostenida; en agosto ya había bajado alrededor de 10 kgs y se agregó sensación febril intermitente, mialgias, diaforesis nocturna y, concomitantemente, disartria, torpeza manual derecha progresiva, diplopía ocasional en miradas vertical ascendente y lateral derecha; un mes después se asociaron adenopatías cervicales.

En octubre del 2005, consultó en el servicio de emergencia del Hospital Barros Luco-Trudeau, por 
cuadro cerebeloso. Se objetivó una presión arterial de 150/110 mmHg, con pulso regular de 88 por minuto, afebril, enflaquecido. Sin lesiones cutáneo-mucosas ni visceromegalias; examen cardiopulmonar normal. Adenopatías cervicales, axilares, inguinales y poplíteas, no dolorosas, adheridas a los planos profundos. Al examen neurológico conservaba conciencia plena y funciones superiores normales. Fondo de ojo normal. Presencia de sacudidas nistágmicas horizontales autolimitadas en el ojo derecho, en mirada extrema ipsilateral. Ataxia de la marcha, mínima hipotonía, dismetría y disdiadococinesia a derecha. Sin signos meníngeos. La TAC cerebral de ingreso evidenció una lesión hipodensa mesencefálica izquierda, que captó contraste en forma nodular. Se hospitalizó con el diagnóstico de probable linfoma sistémico con compromiso del SNC. El hemograma de ingreso no presentó alteraciones, salvo VHS de $33 \mathrm{~mm} / \mathrm{h}$. Hipoalbuminemia (3,1 g/L), con hipergamaglobulinemia (5,6 g/L), corroborada con electroforesis de proteínas. LCR de aspecto límpido, incoloro, acelular, glucosa $0,47 \mathrm{~g} / \mathrm{L}$ (glicemia $76 \mathrm{mg} / \mathrm{dl}$ ) y proteínas de 0,46 g/L, tinción con tinta china, baciloscopia, búsqueda de células neoplásicas y cultivo corriente en LCR negativos. Adenosíndeaminasa (ADA) en LCR 2,1 U/l. Cultivo de Koch en LCR a los 30 y 60 días negativo. La serología para VIH (ELISA) fue positiva. El HBsAg, anticuerpos (ELISA) anti VHC y serología para Chagas fueron negativos, VDRL no reactivo. Sedimentos de orina repetidos mostraron microhematuria, solicitándose baciloscopias de orina. La TAC de abdomen, tórax y pelvis evidenció múltiples adenopatías mediastínicas, retroperitoneales, inguinales y esplenomegalia. La RM cerebral mostró dos lesiones: mesencefálica izquierda de $10 \mathrm{~mm}$ y en el hemisferio cerebeloso izquierdo de $7 \mathrm{~mm}$, ambas con refuerzo anular con gadolíneo (Figura 1).

La evolución fue sin cambios; una semana posterior a su ingreso se le realizó una biopsia ganglionar cervical. El $12^{\circ}$ día de su ingreso se reevaluó la RM cerebral y se inició terapia empírica anti-toxoplasma con pirimetamina $50 \mathrm{mg} /$ día, sulfadiazina 4 gr/día y ácido folínico $15 \mathrm{mg} /$ día. Apareció en su evolución fiebre de hasta $39{ }^{\circ} \mathrm{C}$, manteniéndose la terapia, hasta que por resultado positivo de baciloscopia de orina y de biopsia ganglionar informada como "linfadenitis granulomatosa caseificante con baciloscopia positiva", se modificó el tratamiento a terapia anti-tuberculosa con isoniazida $300 \mathrm{mg} /$ día, rifampicina $600 \mathrm{mg} /$ día, pirazinamida 1.500 mg/día y estreptomicina 750 mg/día. El cultivo de Koch de orina resultó positivo a los 60 días (más de 50 colonias). La serología para toxoplasmosis (IgG e IgM) fue negativa. El recuento de linfocitos CD4 fue de 590 céls $/ \mathrm{mm}^{3}$ (37\%) en dos determinaciones y la carga viral de 56.000 copias de ARN viral/ml. No se realizó PPD. Evolucionó afebril desde el día 13 de tratamiento y fue dado de alta al día 20.

En el examen neurológico a los 30 y 60 días, persistía con disartria y síndrome neocerebeloso derecho, pero desaparecieron la diplopía y el nistagmus del ojo derecho. Permaneció con buena tolerancia y adherencia al tratamiento anti TBC. Hasta el control a los 60 días aún no había iniciado terapia anti-retroviral.

La RM cerebral a las 10 semanas del inicio de la terapia antituberculosa mostró una notable disminución en el tamaño de las lesiones parenquimatosas (Figura 2).
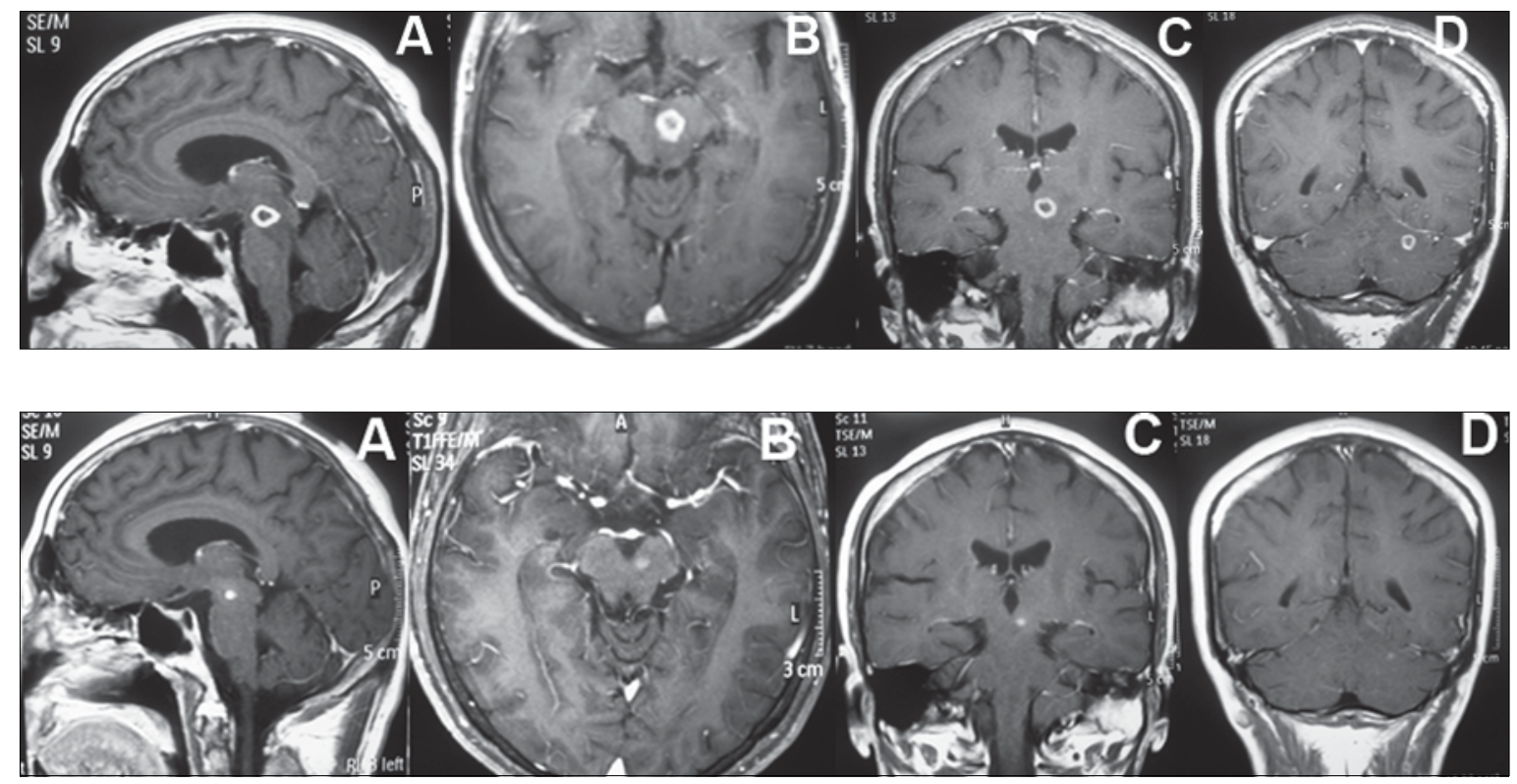

Figura 1. Imagen de RM encefálica, ponderada en T1 con administración de gadolíneo. Muestra lesiones mesencefálica (A, B y C) y cerebelosa (D) izquierdas que se realzan en forma anular.

Figura 2. Imágenes de control posterior a 10 semanas de tratamiento anti TBC. La secuencia mostrada es homóloga a la figura 1, y evidencia una franca disminución en el tamaño y realce lesional con gadolíneo. 


\section{Discusión}

Entre 5 y 15\% de las TBC extra-pulmonares corresponden a compromiso del SNC ${ }^{3,4}$ (Tabla 1) y la forma de presentación más frecuente es la meningitis tuberculosa $^{8,9}$. Las formas parenquimatosas cerebrales son raras, siendo más frecuentes en pacientes infectados con VIH, especialmente los tuberculomas, que pueden ocurrir con o sin meningitis ${ }^{7}$. Éstos se manifiestan clínicamente como lesiones ocupantes de espacio ${ }^{4}$, y su presentación es más arrastrada que la meningitis $\mathrm{TBC}^{7}$. Los hallazgos clínicos están determinados por su localización, predominando la infratentorial en niños y supratentorial en adultos. Se ubican preferentemente en las regiones frontal y parietal; se describen también en la región selar, tronco cerebral, tálamo,

\begin{tabular}{l} 
Tabla 1. Tuberculosis del SNC \\
Meningitis tuberculosa \\
Leptomeníngea \\
Paquimeníngea \\
TBC parenquimatosa cerebral \\
Tuberculomas parenquimatosos \\
TBC cerebral miliar \\
Absceso tuberculoso \\
Cerebritis focal tuberculosa \\
Encefalopatía tuberculosa \\
TBC medular \\
Radiculomielitis tuberculosa \\
Tuberculoma medular \\
Compromiso medular extrínseco (absceso vertebral) \\
\hline
\end{tabular}

Tabla 2. Características de masas encefálicas tuberculosas en la tomografía axial computada y en la resonancia magnética (modificada de ref. 5)

\begin{tabular}{lll|} 
& TAC & RM \\
\hline Granuloma no caseoso & SC: hipo/isodenso & T1: hipointenso \\
& CC: realce homogéneo & T2: hiperintenso \\
& & T1-Gd: realce homogéneo \\
Granuloma caseoso & CC: realce heterogéneo & T1: hipo-intermedia señal \\
con centro sólido & central; realce anular & T2: intermedia-hipo señal \\
Granuloma caseoso & SC: hipodenso & T1-Gd: realce anular \\
con centro líquido & CC: realce anular & T1: hipointenso \\
& & T2: hiperintenso \\
\hline SC: $\sin$ contraste; CC: con contraste & T1-Gd: realce anular \\
\hline
\end{tabular}

ganglios basales, ángulo ponto-cerebeloso, vía óptica, región pineal, ventrículos, médula espinal y a nivel subdural, epidural e intradural extramedular ${ }^{3-5,7,10-12}$. Los síntomas y signos más comunes son cefalea, convulsiones, déficit neurológico focal, edema de papila y síntomas de hipertensión endocraneana. Ocasionalmente puede existir fiebre ${ }^{7}$. Las alteraciones licuorales son infrecuentes, lo más común es una discreta hiperproteinorraquia inespecífica.

El cultivo de Koch es usualmente negativo. De mayor importancia en el diagnóstico es la neuroimagen y la respuesta a la terapia anti $\mathrm{TBC}^{7}$. En la Tabla 2 se mencionan las características imagenológicas de las lesiones parenquimatosas focales tuberculosas del SNC. En casos donde no es posible realizar el diagnóstico de TBC a partir de una localización fuera del SNC, puede requerirse una biopsia cerebral ${ }^{4}$.

La TBC del SNC puede producirse durante la infección primaria o reactivación ${ }^{4}$ y siempre es secundaria a un foco primario extra-neurológico ${ }^{13}$.

Según la OMS, la TBC causa la muerte de $11 \%$ de los pacientes con SIDA. Otros grupos de riesgo son drogadictos endovenosos, inmigrantes de zonas de alta prevalencia y población en hacinamiento ${ }^{14}$.

La TBC en pacientes con infección por VIH ocurre con cualquier nivel de recuento de LT CD4, siendo más frecuente la forma de TBC extra-pulmonar o diseminada con LT CD4 bajo 200 céls/mm ${ }^{3}{ }^{14}$.

En pacientes con infección por VIH con masas cerebrales, una vez excluida una toxoplamosis cerebral, aún en ausencia de TBC sistémica demostrable, se debiera considerar el inicio de tratamiento anti TBC en zonas con alta prevalencia de $\mathrm{TBC}^{4,15}$. En nuestro paciente se detectó la presencia de bacilos de Koch en orina y en linfonodos, orientando el diagnóstico e inicio de terapia específica.

En el caso expuesto las manifestaciones clínicas y neurológicas orientaban a un compromiso infratentorial (cerebeloso y mesencefálico), lo que fuera corroborado posteriormente con la neuroimagen. A la dificultad diagnóstica etiológica contribuyó el desconocimiento de que padecía de una TBC sistémica y que tenía además una infección por VIH/SIDA. Una particularidad fue la normalidad en todos los parámetros del LCR, inclusive el valor de ADA, lo que retrasó el diagnóstico.

La inmunosupresión por VIH es el al factor de riesgo más importante para la progresión de una TBC latente a activa ${ }^{16}$. En más de la mitad de los casos es extrapulmonar ${ }^{17}$, como en nuestro paciente, en quien se demostró una TBC renal y ganglionar, además de compromiso del SNC. La incidencia de TBC del SNC alcanza hasta $10 \%$ en pacientes con infección por VIH versus tuberculosos sin infección por $\mathrm{VIH}^{17}$. 
La forma clínica más frecuente de TBC del SNC en pacientes infectados por $\mathrm{VIH}$ es la meningitis ${ }^{17}$, pero en nuestro caso la presencia de tuberculomas fue sin compromiso meníngeo. En estos pacientes no se alteraría la presentación clínica de la meningitis $\mathrm{TBC}^{4}$, pero existen diferencias comparativas entre grupos con y sin infección por VIH, como son: baja frecuencia de meningismo (17\%), mayor frecuencia de adenopatías y masas intracraneales y baja incidencia de parálisis de nervios craneales (6\%) en los pacientes seropositvos ${ }^{17}$.

La mortalidad durante el tratamiento por TBC en general y en TBC meníngea es mayor en pacientes con SIDA $^{9,16}$. Son factores de mal pronóstico la demora mayor a dos semanas en instaurar la terapia específica desde el inicio de los síntomas y un recuento de LT CD4 menor a 200 céls/ $\mathrm{mm}^{3}{ }^{17}$. A diferencia de los no portadores de $\mathrm{VIH}$, el uso de corticosteroides no afecta el pronóstico ${ }^{18}$.

En la evaluación de pacientes con SIDA y lesiones intracraneales debe considerarse el grado de inmunosupresión. Con recuentos de LT CD4 mayor a 500 céls/ $\mathrm{mm}^{3}$ las causas son similares a la población inmunocompetente: tumores primarios y metástasis, cuyas neuro-imágenes pueden ser similares a los tuberculomas. Con recuentos de LT CD4 menor a 200 céls $/ \mathrm{mm}^{3}$, son más frecuentes las infecciones oportunistas y los tumores asociados al SIDA. Encontramos infecciones oportunistas causadas por parásitos (toxoplasmosis, neuro-cisticicercosis), hongos (Cryptococcus neoformans, Candida albicans, Aspergillus sp, Mucor sp) y bacterias ( $M$. tuberculosis y $M$. avium intracellulare, Nocardia sp, Treponema pallidum). Los tumores más frecuentes son el linfoma primario del SNC, los gliomas y metástasis. También debe considerarse en el diag- nóstico diferencial la enfermedad cerebrovascular ${ }^{18-21}$.

Creemos importante comunicar este caso por corresponder a un escenario clínico poco habitual de TBC del SNC, siendo las manifestaciones neurológicas el motivo de consulta, el estudio de LCR fue normal y las lesiones visualizadas en la RM cerebral inespecíficas.

Dado que en nuestro país la TBC es prevalente y la infección por VIH va en aumento, la TBC debe ser considerada siempre en el diagnóstico diferencial de masas cerebrales en estudio, independiente de la condición de portador de VIH y del recuento de LT CD4.

\section{Resumen}

Presentamos el caso clínico de un paciente que consultó por un síndrome cerebeloso y diplopía, asociados a pérdida de peso de 10 kilos en 6 meses y adenopatías múltiples. El estudio imagenológico cerebral demostró la presencia de lesiones de aspecto granulomatoso a nivel cerebeloso y mesencefálico, sin alteración citoquímica del líquido cefalorraquídeo. Las baciloscopias de orina y de la biopsia ganglionar fueron positivas y permitieron el diagnóstico de tuberculosis (TBC) sistémica. La serología para VIH resultó positiva en sangre con un recuento de linfocitos CD4 de $590 \mathrm{cel} / \mathrm{mm} 3$, clasificándose el caso como SIDA etapa C1. El paciente fue tratado con el esquema antituberculoso según norma nacional, con buena respuesta clínica e imagenológica. La presentación de tuberculomas infratentoriales sin meningitis, como primera causa de pesquisa de TBC y SIDA no es habitual, de allí nuestro interés por comunicar el caso.

\section{Referencias}

1.- Global Tuberculosis Control: Surveillance, Planning, Financing. WHO Report 2005, Geneve, World Health Organization.

2.- Frieden T, Sterling T, Munsiff S, Watt C, Dye C. Tuberculosis. Lancet 2003; 362: 887-99.

3.- Katti M K. Pathogenesis, diagnosis, treatment, and outcome aspects of cerebral tuberculosis. Med Sci Monit 2004; 10 (9): 215-29.

4.- Verma A, Solbrig M. Infecciones del Sistema Nervioso: Infecciones Bacterianas. En: Bradley W, Daroff R, Fenichel G, Jankovic J. Neurología Clínica. $4^{\circ}$ edición. Madrid, España. Elsevier. 2005; 1455-1495

5.- Wolff M. Cambios epidemiológicos en las enfermedades infecciosas en Chile durante la década 1990-2000. Rev Méd Chile 2002; 130: 353-62.

6.- Estadísticas Enfermedades de notificación obligatoria. Ministerio de Salud, Chile. Disponibles en URL: www.minsal.cl. (accedido 28 noviembre 2006).

7.- Bernaerts A, Vanhoenacker F M, Parizel P M, Van Goethem J W, Van Altena R. Tuberculosis of the central nervous system: overview of neuroradiological findings. Eur Radiol 2003; 13: 1876-90.

8.- Enberg M, Quezada M, De Toro C, Fuenzalida L. Meningitis tuberculosa en adultos: Análisis de 53 casos. Rev Chil Infect 2006; 23: 134-9.

9.- Thwaites G E, Tran T H. Tuberculous meningitis: many questions, too few answers. Lancet Neurol 2005; 4: 160-70.

10. - Minagar A, Schatz N J, Glaser J S. Case report: one-and-a-half-syndrome and tuberculosis of the pons in a patient with AIDS. AIDS Patient Care STDS 2000; 14: 461-4.

11.- Kemalogïu S, Gür A, Nas K, Çevik R, Büyükbayram H, Saraç A. Intramedullary tuberculoma of the conus medullaris: case report and review of the literature. Spinal Cord 2001; 39: 498-501.

12.- Roca B. Intradural extramedullary tuberculoma of the spinal cord: a review of reported cases. J Infect 2005; 50: 425-31.

13.- Sáez-Méndez D, Nogales-Gaete J. Meningitis Crónicas. En: Nogales-Gaete J, Donoso A, Verdugo R. Editores Tratado de Neurología Clínica. Santiago: Editorial Universitaria, 2005: 345-350.

14.- Benson C, Kaplan J. Treating opportunistic 
infections among HIV infected adults and adolescents: Recommendations from CDC, the National Institutes of Health, and the HIV Medicine Association/Infectious Diseases Society of America. Clin Infect Dis 2005; 40: S131-235.

15.- Del Brutto O, Mosquera A. Brainstem tuberculoma mimicking glioma: The role of antituberculous drugs as a diagnostic tool. Neurology 1999; 52 (1): 210-11.

16.- Burman W. Issues in the management of HIV-related tuberculosis. Clin Chest Med 2005; 26: 283-94
17.- Sánchez-Portocarrero J, Pérez-Cecilia E, Romero-Vivas J. Infection of the centra nervous system by Mycobacterium tuberculosis in patients infected with human immunodeficiency virus (the new neurotuberculosis). Infection 1999; 27: 313-17.

18.- Prasad K, Volmink J, Menon G. El uso de esteroides en el tratamiento de la meningitis tuberculosa (Revisión Cochrane traducida). En: La Biblioteca Cochrane Plus, 2005 Número 4, Oxford: Update Software Ltd. Disponible en http: //www.update- software.com. (accedido 20 noviembre 2006).

19.- Manji H, Miller R. The neurology of HIV infection. J. Neurol Neurosurg Psychiatry 2004; 75: 29-35.

20.- Bayindir C, Mete O, Bilgic B. Retrospective study of 23 pathologically proven cases of central nervous system tuberculomas. Clin Neurol Neurosurg 2006; 108 (4): 353-7.

21.- Koralnik I. Approach to HIV-infected patients with central nervous system lesions. Up to date. Disponible en URL: www. uptodate.com. (accedido 11 octubre 2007). 